\title{
Probing System Characteristics in Coordinate Metrology
}

\author{
Salah H. R. Ali \\ Engineering and Surface Metrology Lab, Length and Precision Engineering Division, \\ National Institute for Standards (NIS), Giza (12211), PO Box 136, Egypt, Dr_Salah@nis.sci.eg
}

\begin{abstract}
This paper aims at studying the effect of the dynamic errors on surface measurements using three different types of touch trigger probes attached to a bridge-type-CMM. Unforeseeable dynamic root errors of a ductile touch trigger probing system have been characterized theoretically and experimentally as well. The results were employed in validating a developed analytical twodimensional-model (2DM) of stylus tip to be developed to demonstrate the capability of such approaches of emphasizing the root error concept, and to evaluate the accuracy of the CMM measurements. A set of experiments was conducted; the results were analyzed in order to investigate the effect of the dynamic root errors in the light of probe scanning speed at different stylus tip radii. Variations in the mass and geometry of the stylus have their consequent effects on its inherent intrinsic dynamic characteristics that in turn would cause relevant systematic root errors in the resulting measurements. 3D bore cylindrical surface form undulations were measured by employing a probe on the trajectory of internal surface diameter for the standard reference test gauge ring. Regression analysis was applied on the results of measurement density distribution; uncertainty of measurement repeatability was then evaluated and graphically presented. The results were investigated and optimum strategic measurement parameters could thus have been derived to ensure foreseeable accurate and precise results.
\end{abstract}

Keywords: Trigger probe, tip radius, 2DM, dynamic errors, surface undulations, and CMM accuracy.

\section{INTRODUCTION}

Cwic MM (COORDINATE MEASURING MACHINE) is widely utilized as a precise dimensional measuring tool in the modern manufacturing processes, especially in the mass production, as in automotive industries.

CMM probe is one of the most important systems of dimensional measuring instruments and responsible for the coordinate measurement accuracy [1]-[5]. Fast response and accurate detection of the probe that can be computer controlled, represents the current trend for the next generation of coordinate metrology. Varieties of probe designs are already available and compatible with most of the CMMs [6]. The probing system in CMM machines includes stylus and stylus tip which have their own dynamic characteristics during the measuring process [7]. The stylus tip contact with the detected surface is the source of signals that will develop the pattern on the working objects. So, the performance of the CMM overall system is very much dictated by the motion precision of the probe tip and its actuator. Therefore, the probe stylus tip is laterally at the center of the CMM operation and a key element of coordinate measurements. The detection probes branch into two main categories; there are contact (tactile) probes and non-contact probes. The contact probe gathers data by physically touching the specimen directly, which can be classified into two specific families of manual hard probes and touch trigger probes [8]-[9].

\subsection{Hard Probe}

The hard probes are available in a variety of configurations and continue to have a broad application in coordinate metrology. This type of probes is used with manual CMMs for low and medium accuracy requirements. Hard probes are simple in use and rugged too, but their repeatability quality depends upon their operator touch.
Because every operator has a different touch when moving and bringing the probe into contact with the feature, this hard type probe is not commonly used in large mass production, which requires high level accuracy.

\subsection{Trigger Probe}

Touch trigger probe is the recent commonly used type in $\mathrm{CMM}$, it has a precision-built-in and touch-sensitive device that generate an electronic signal through probe tip contact with the specimen surface, which is usually indicated as visual LED light and an audible touch signal. The probe head itself is mounted at the end of one of the CMM's moving axes, it can be rotated automatically, and many different probe stylus tips can be accommodated and attached. These capabilities make the CMM trigger probe a versatile and flexible data-gathering device. CMM equipped with trigger probes eliminates the influence of operator touch on the quality of measured data compared to the hard probe type, because the trigger probe can be fitted on direct computer numerical control (CNC-CMMs) and manual CMMs systems [8], [10]. An additive improvement to the basic touch trigger probe design includes a piezoelectricbased sensing to transmit the deflection of the probe into a constant digital acoustic signal that is recorded by the CMM. This design ensures high rate of measurement accuracy in accordance with the elimination of the stylus bending behavior and the probe's internal electromechanical reactions.

Theoretical analysis and experimental studies recently spotted the main source of probe detection errors [1]-[7], [11]-[23] on the touching point . Practically, measurement accuracy is determined by the kinematic accuracy of the $\mathrm{CMM}$ probe and a big portion of used machines. Some of these researches studied CMM error combination of coordinate frames as a moving rigid body of the measuring volume and numerical error compensation with different 
mapping methods, the effect of geometrical errors leading to accuracy improvement [6]-[7]. Considerable research works have been reported to improve the kinematic accuracy of the CMM probe, which is too sophisticated to implement. Few programs focus on changing the $\mathrm{CNC}$ program to compensate the probe error [4], [7], [14], waviness deviation of the measured mechanical parts is the desired value owing to many quasi-static systematic errors as inherited intrinsic geometric errors of probe tip, thermally induced distortions of machine probe elements, probe stiffness, touch force and some error sources [1]-[2], [5]-[7]. Software-based error compensation is a method for anticipating the effect combination of all given factors on standard ring accuracy and suitably modifying the conventionally designed probe tip scanning trajectory. A compensation model has been developed to clear the parametric study effect on the dynamic measurement errors [20], [22], [23]. Other experimental works have been performed to study the scanning measuring machine. Some of these researches analyzed the effects of scanning speed on the performance of different CMMs to evaluate the measurement performance within special conditions on CMM measurements [22]-[23]. However, most of these studies could not separate the performance of the probing system from other error sources through probe scanning. Approaches to derive the dynamic effects of CMM probing systems and techniques of probing compensation have been studied [14], [19]-[22]. Moreover, the stylus tip of the triggering system is limited by its dynamic root errors that may markedly affect its response characteristics [17]-[18]. So, many researchers have focused on studying the CMM trigger probe characteristics. CMM vibrations in the time domain have been discussed to insure the Monte Carlo methods as a generally used tool for understanding dynamic problem resolution [21]. Significant work towards the influence of the object material stiffness, surface shape and its roughness based on measurements of the distance between reference and triggering points in various directions by the rotation of a precise rotary table was performed [2]. That work shows the influence of the specimen material stiffness on pretravel variation depending on measuring force settings as statistically significant. It has been shown that specimen surface roughness has also significant influence on the touch trigger probe pretravel variation independently on applied measuring force [2]. Another approach for stylus tip radius correction has been based on the use of the part CAD model using straight line measurement [3]. Some other researchers studied the influence of the actual configuration of the probe length and volume on the accessibility of inner features, such as slots and holes [5]. Dynamic analysis of a simple mechanical model of touch trigger probe including the effects of stylus bending and the frictional interaction between the stylus ball and the part surface during applied force has been researched [19]. However, in the present work, the stylus tip envelop method proposed to define the measured waviness profile, and discuss the error resulting from the probe tip angle due to rotation with the real circumference surface contact during scanning at different tip sizes. On the other hand, generating profile of cylindrical surface often concavely deviates from the ideal straight line surface, it especially initiating the necessity of measurement during the measuring process. In the difficult example, CMM metrology machine is used to measure the amount of deviation in the roundness waviness to determine the measurement errors using a suitable measurement strategy similar to probe styli characteristics and a suitable standard ring gauge according to the ISO 10360-6:2009 [7], [24]. More efforts are required for static and dynamic stylus response analyses to classify and evaluate the measurement error sources according to the considered design parameters, especially for the construction of new CMMs [22]-[25].

In this paper, three parameters affecting the measurement errors are considered. The first parameter is the rotation angle of probe stylus tip; the second one is the stylus tip size as root of unforeseeable errors. The third parameter is the speed of the CMM touch trigger probe. These measurements are determined accurately using reference artifact including concavely cylindrical surface. Finally, a novel method to verify the dynamic characteristics of the probing system is proposed theoretically and experimentally in order to improve the quality of CMM measurement accuracy.

\section{ANALYTICAL MODEL}

Since the influence of some unforeseeable factors affecting probe inaccuracy could be small, it requires an accurate mathematical model during analysis. Thus, for this investigation a new two-dimensional-model (2DM) has been used to present the root dynamic errors due to ball tip size of the CMM probe stylus at measurement operations.

\subsection{CMM Probe Ball Tip Error}

Throughout scanning, all touch probes in the CMM coordinate measurement have natural ball tip errors [14], [17]-[18]. We have developed 2DM, where the stylus ball is steadily placed in a horizontal position, thus only $\mathrm{X}$-axis and $\mathrm{Y}$-axis translation movement of the stylus is possible. Assuming no stylus tip ball deformation and no surface deformation under the test [17]-[18], the designed 2DM model can be presented in Figs. 1 and 2.

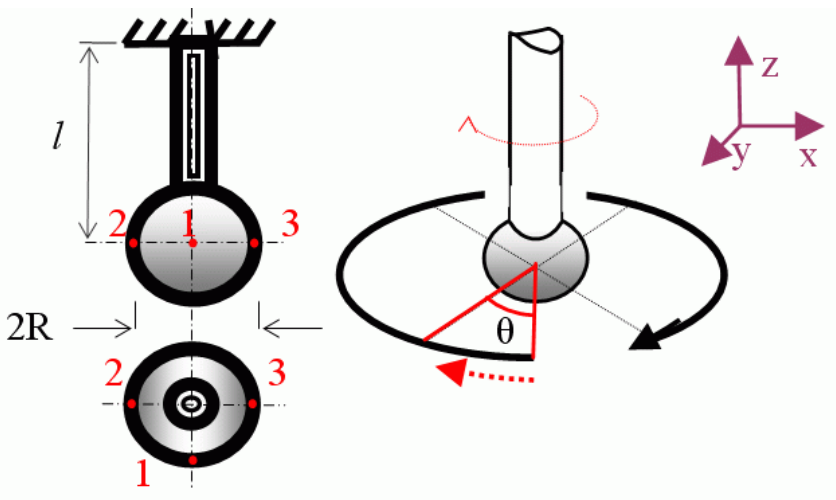

Fig.1. Horizontally placed probe stylus ball tip radius (R).

The measurement principle of the proposed system includes contact points 1,2 and 3 that are indicated on the vertical and horizontal plans of the probe stylus tip with $l$ stylus length and ball tip radius R, as showing in Fig.1. Fig.2 
shows that due to the finite size of the probe stylus ball tip, the contact point on a cylindrical surface will be along the stylus axis, but alternatively at some point on the side of the ball where the test surface and the stylus tip ball slope match horizontally. Because the ball does not touch the test artifact specimen along the same stylus slope angle, there will be an error $\mathrm{E}$ in the measured length for any measurement point where the test part surface slope $(\theta)$ is not zero degree. The error $\mathrm{E}$ and different possible positions of the ball tip are shown. Case $d$ is at a higher surface slope $\left(180^{\circ}\right)$ and thus has a larger measurement error $\mathrm{E}$, while case $\mathrm{a}$ is located at a lower surface slope and has a smaller measurement error E. Therefore, to get exact location of $\mathrm{n}$ point on slope surface, an error $\mathrm{E}$, reduced by value $\Delta \mathrm{Y}$ is made, due to fact, that position of point $t$ on stylus tip ball is captured every time. From Fig.2, values $\Delta \mathrm{Y}$ and $\mathrm{E}$ can be expressed as follows:

$$
\begin{gathered}
\Delta \mathrm{Y}=\mathrm{R}-\mathrm{r} \\
\Delta \mathrm{Y}=\mathrm{R}-(\mathrm{R} \cos \theta)=\mathrm{R}(1-\cos \theta)
\end{gathered}
$$

Where the distance between two points $\mathrm{t}$ and $\mathrm{n}$ indicates the $\mathrm{E}$ in $\mathrm{Y}$ direction, while $\Delta \mathrm{Y}$ is the relative distance between points $\mathrm{m}$ and $\mathrm{t}$ in the $\mathrm{Y}$ direction, the large scale of the tip ball in case b. From appointed 2DM can be stated that measurement error $\mathrm{E}$ and $\Delta \mathrm{Y}$ values are made only on $\mathrm{Y}$-axis and are dependent on surface slope. In point 2 or 3 (according to Fig.1, where matching angle is $\theta=180^{\circ}$ ) $\Delta \mathrm{Y}$ and error $E$ would be maximal values, while error $E$ and $\Delta Y$ are equal to zero only at scanning flat surfaces $\left(\theta=0,360^{\circ}\right)$ when all points $m, n$ and $t$ are overlaying each other $(m=n=t)$ as shown in the case a.

\subsection{Results of Analytical Model}

The root error due to CMM stylus ball tip can not be neglected. Three different stylus balls with-radii $\mathrm{R}=4.0,2.5$, and $1.5 \mathrm{~mm}$ have been chosen. A relative mutation in the $\mathrm{Y}$ direction $(\Delta \mathrm{Y})$ of the stylus ball can be observed according to the surface slope degree that is called matching angle $(\theta)$, Fig.3.

In the course of the application of accurate analytical 2DM proposed in the measurement of cylindrical parts using CMMs, emerged two types of systematic unforeseeable errors. The first error resulted from increasing the surface slope angle while the second error resulted from increasing the radius of the stylus tip ball. Fig. 3 shows how margin of errors are calculated theoretically and the output of climate surface slope degree $(\theta)$ of the probe tip during contact through $360^{\circ}$ (using complete cylindrical reference artifact) for different three tips radii. It is observed that the amount of errors in the Y-direction starting from zero for each probe tip at the beginning of contact at $0^{\circ}$, while increasing incremental increase of inclination angle of a point contact of the tip with the artifact reaches its maximum value of $2 \mathrm{R} \%$ at $180^{\circ}$ and then comes back to the decline to reach zero at $360^{\circ}$. It means that rotational motion that occurs during the probe tip scanning due to creeping of the tip at the base of the probe vibrates at the surface coming into contact with the cylindrical parts which are also generating another error regularly.
From the theoretical work presented in this paper, it seems that the results of root errors caused by working mechanism of the touch trigger probe are relatively small. Thus, at the confluence or collection of these errors with other dynamic errors in the measurement, the error values have been tremendously increased and they will be discussed experimentally in the following sections.

\section{EXPERIMENTAL WORK}

The structure of the bridge-type-CMM in this study is a typical CMM ductile touch-trigger-scanning probe in the national institute for standards (NIS) at Giza, Egypt. The driving system of $Y$ is located on the right side of the bridge. The bridge travels along the $Y$-guide way, the $Y$-carriage travels along the $X$-traverse and the ram together with the probe travel along the $X, Y$ and $Z$-directions. ZEISS-BridgeType-CMM was equipped with PRISMO-VAST touchtrigger probe head under different direct computer control parameters $[3,25]$. In the measurement process, on a $\mathrm{CNC}$ $\mathrm{CMM}$ the probe is commanded to approach the artifact at a constant speed (positioning velocity) when it comes within the probe approach distance. We prepared experimental work based on a four steps set. This set is prepared to verify the CMM machine according to ISO to be suitable for implementation of the schedule of parametric study measurements using experimental set up to achieve the goal of this research.

\subsection{Verification of CMM Stylus System}

Measuring of the waviness of the $200 \mathrm{~mm}$ standard artifact gauge ring using CMM has been conducted at standard conditions. The ambient environmental conditions during the experiments in the laboratory were recorded. The room temperature was maintained within the range $20.0 \pm 0.5^{\circ} \mathrm{C}$; while the humidity was maintained at $50 \pm 2 \%$. The standard ISO 10360-6:2009 [7], [24] specifying the procedure by Gaussian (Least-Square) for assessment software and probing system has been verified, Table 1 .

Table 1 Results of probing error thought verification scanning.

\begin{tabular}{|l|c|c|}
\hline \multicolumn{1}{|c|}{ CMM element } & $\begin{array}{c}\text { Measured radius, } \\
\mathrm{mm}\end{array}$ & $\begin{array}{c}\text { Standard deviation } \\
\mathrm{S}_{\mathrm{D}}, \mathrm{mm}\end{array}$ \\
\hline Master probe & 3.9998 & 0.0001 \\
\hline Reference sphere & 14.9942 & 0.0001 \\
\hline $1^{\text {st }}$ used probe & 4.0001 & 0.0001 \\
\hline $2^{\text {nd }}$ used probe & 2.5003 & 0.0001 \\
\hline $3^{\text {rd }}$ used probe & 1.4992 & 0.0001 \\
\hline
\end{tabular}

The maximum permissible measurement error $\left(\mathrm{MPE}_{\mathrm{E}}\right)$ for the used CMM machine evaluated according to ISO 10360$2[3,26]$; the maximum permissible probing error $\left(\mathrm{MPE}_{\mathrm{P}}\right)$ and the maximum permissible tangential scanning probing error $\left(\mathrm{MPE}_{\mathrm{Tij}}\right)$ [3], [4], [10], [13], [24] are defined as follows:

$$
\mathrm{MPE}_{\mathrm{E}}=\mathrm{A}+\mathrm{L} / \mathrm{K}, \mathrm{MPE}_{\mathrm{P}}= \pm 1.0 \mu \mathrm{m} ; \mathrm{MPE}_{\mathrm{Tij}}= \pm 1.9 \mu \mathrm{m}
$$

Where $\mathrm{A}$ is a constant value equal to $0.9 \mu \mathrm{m}, \mathrm{L}$ is the measuring length in $\mathrm{mm}$, and $\mathrm{K}$ is the $\mathrm{CMM}$ manufacture constant equal to 350 . 


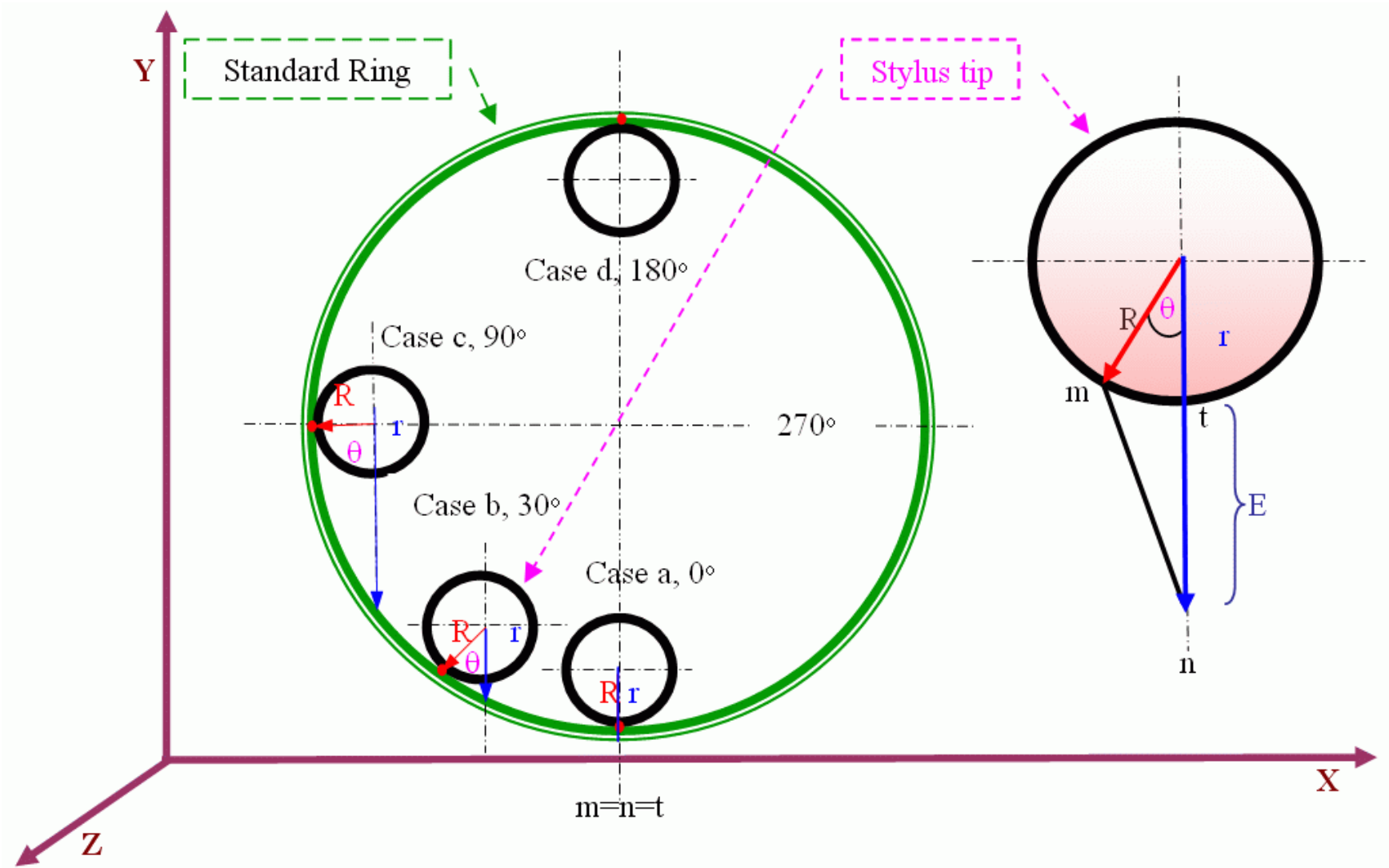

Fig.2. Size of error E related to the probe tip ball radius (R) and surface slope degree $(\theta)$.

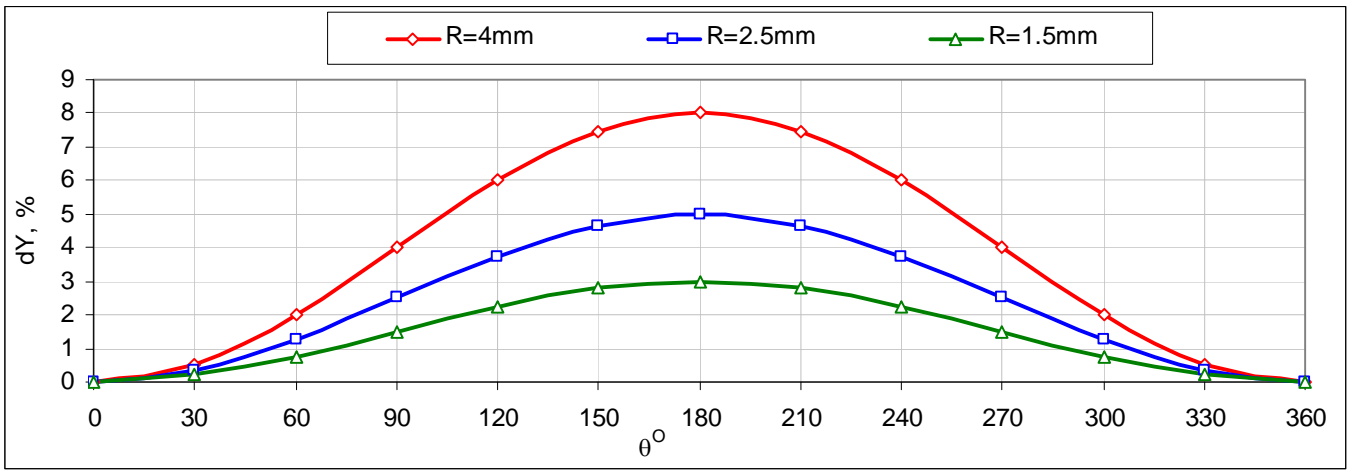

Fig.3. Relative error of the ball tip at different surface slope angles.

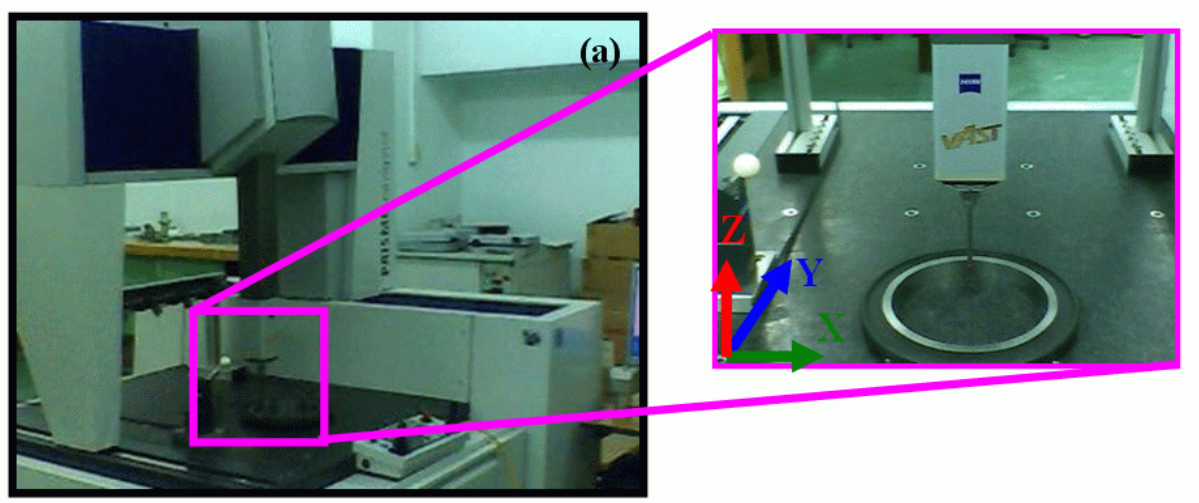

(b)

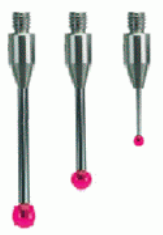

Fig.4. Experimental setup using (a) ZEISS-Bridge-Type-CMM, and (b) trigger probe styli with spherical ruby tips. 


\subsection{Experimental Procedure}

To demonstrate the feasibility of the proposed probe stylus tip size after envelop, verification methods for corrected measured point determination were carried out on a movable bridge ZEISS PRISMO CMM equipped with VAST scanning probe head of $0.01 \mu \mathrm{m}$ resolution. The main task of a probing system is to detect whether the tip is in contact with the artifact and to provide a feedback signal to the CMM control so that all motion from the axes is stopped and the positions of the measured axes can be determined. Experiments were carried out for three different stage types of probe styli. 3D surfaces of the probe ball tip pretravel were collected using standard test $200 \mathrm{~mm}$ ring as an artifact at different scanning speeds with 600 points per scanning track while the traveling speed was $50 \mathrm{~mm} / \mathrm{s}$ with Gaussian software fitting technique. The probe stylus tip was in contact with the inner circumference surface of the standard gauge artifact to set the origin of the working coordinate system as shown in Fig.4a. Actually, the center of probe tip is the center of measure. A complete schematic of the instrument setup procedures is shown in Fig. 4 for three different probe styli with spherical red ruby tips, see Fig.4b. All values of the design parameters of these experiments are mentioned in Table 2.

Table 2 Schedule of the parametric study of three work groups.

\begin{tabular}{|c|c|c|c|c|}
\hline \multirow{2}{*}{$\begin{array}{l}\text { Test } \\
\text { no. }\end{array}$} & \multicolumn{3}{|c|}{ Probe stylus specifications } & \multirow{2}{*}{$\begin{array}{c}\text { Probe tip } \\
\text { scanning } \\
\text { speed, } \mathrm{mm} / \mathrm{s}\end{array}$} \\
\hline & $\begin{array}{l}\text { Tip radios, } \\
\mathrm{mm}\end{array}$ & $\begin{array}{c}\text { Mass, } \\
\mathrm{g}\end{array}$ & $\begin{array}{c}\text { Length, } \\
\text { mm }\end{array}$ & \\
\hline 1 & \multirow{6}{*}{4.0} & \multirow{6}{*}{26.4} & \multirow{6}{*}{63.5} & 5 \\
\hline 2 & & & & 10 \\
\hline 3 & & & & 15 \\
\hline 4 & & & & 20 \\
\hline 5 & & & & 25 \\
\hline 6 & & & & 30 \\
\hline 7 & \multirow{6}{*}{2.5} & \multirow{6}{*}{9.2} & \multirow{6}{*}{53.0} & 5 \\
\hline 8 & & & & 10 \\
\hline 9 & & & & 15 \\
\hline 10 & & & & 20 \\
\hline 11 & & & & 25 \\
\hline 12 & & & & 30 \\
\hline 13 & \multirow{6}{*}{1.5} & \multirow{6}{*}{4.5} & \multirow{6}{*}{33.5} & 5 \\
\hline 14 & & & & 10 \\
\hline 15 & & & & 15 \\
\hline 16 & & & & 20 \\
\hline 17 & & & & 25 \\
\hline 18 & & & & 30 \\
\hline
\end{tabular}

\subsection{Parametric Study of Stylus Design}

The parameters of the study are tabulated, based on three different groups $\mathrm{a}, \mathrm{b}$ and $\mathrm{c}$ as shown in Table 2. The measurements were conducted through 90 individual tests including five repeating times for different scanning speeds, with three different specifications of CMM probe stylus.

\subsection{Measurement Density}

For the CMM probe styli repeatability tests of measurements, a reference standard artifact ring was fixed on the surface of Garnet flat table of CMM as illustrated in Fig.4. The probe head was then rotated through $365^{\circ}$ with an interval of $0.01 \mathrm{~mm}$ during the circumference of the selected standard gauge ring. The collected data were used to determine the standard deviation for each indexing speed at different probe tip radii using statistical analysis. The variations of form or waviness measurements for the triggered probes were plotted in Figs.5-7 to predicate the density of the deviations due to the repeatability of measurements.

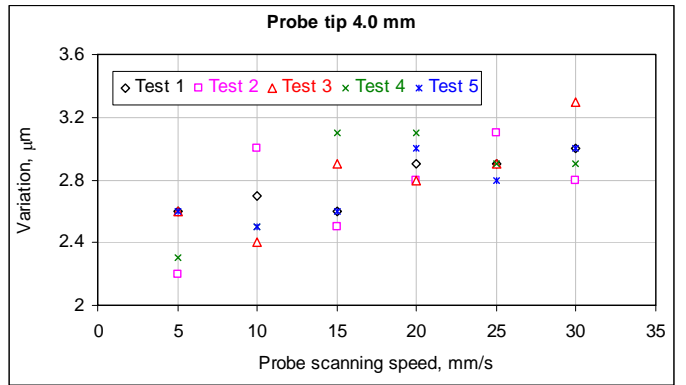

Fig.5. Roundness variations for $4.0 \mathrm{~mm}$ stylus tip radius size at different scanning speeds.

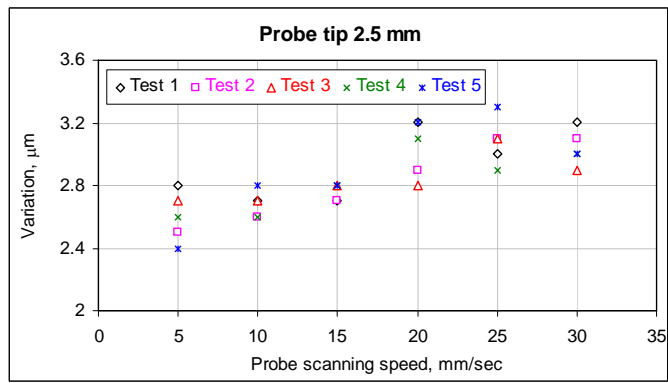

Fig.6. Variations of roundness for $2.5 \mathrm{~mm}$ stylus tip radius size at different scanning speeds.

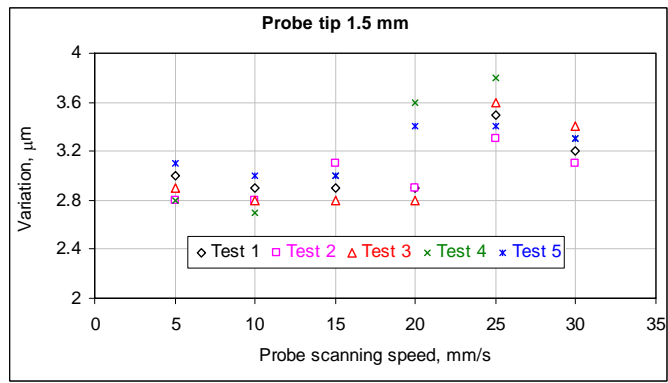

Fig.7. Roundness variations at different scanning speeds for stylus tip radius of $1.5 \mathrm{~mm}$.

\subsubsection{Stylus tip size $4.0 \mathrm{~mm}$}

Fig.5 presents the results from the measurement repeatability of a touch trigger probe with a $4.0 \mathrm{~mm}$ stylus tip radius using the test rig. As can be seen in the figure, the repeatability varies for different approach directions of about $1.1 \mu \mathrm{m}$ averaged value. Each color (five colors) in the graph in this figure represents a standard deviation for thirty runs at mentioned scanning speeds, Table 2 . To establish the number of the required repeated runs, test signals were carried out to observe the data variations related to an 
increasing number of measurements. Based on the results, the number of repeated runs was selected to be five, since no significant difference existed between these measurements higher than $0.6 \mu \mathrm{m}$.

\subsubsection{Stylus tip size $2.5 \mathrm{~mm}$}

The presentation of results in Fig. 6 shows the density of measured points for roundness waviness variation at different scanning speeds for the same conditions. Once again, the tests were conducted at different approach directions using the same stylus tip of $2.5 \mathrm{~mm}$ for the same probe head. These results stated that the probe repeatability should be within $0.4,0.2,0.1,0.4,0.4$, and $0.3 \mu \mathrm{m}$ for 5,10 , $15,20,25$, and $30 \mathrm{~mm} / \mathrm{s}$ probe tip scanning speed respectively.

\subsubsection{Stylus tip size $1.5 \mathrm{~mm}$}

For the same conditions, the repeatability error increases dramatically when longer styli are used. Fig. 7 shows that the repeatability is adversely affected by decreasing stylus tip. Once again, the tests were conducted at different approach directions using the probe stylus tip radius of $1.5 \mathrm{~mm}$ with the same CMM probe head. These results stated that the probe repeatability should be within $0.3,0.3,0.35,0.8,0.4$, and $0.3 \mu \mathrm{m}$ for $5, \ldots$, and $30 \mathrm{~mm} / \mathrm{s}$ scanning probe tip speeds. From results in Figs.5, 6, and 7 becomes evident that:

- Detected deviations increase when increasing the speed of measurements for each stylus tip radius $4.0,2.5$, or 1.5 $\mathrm{mm}$.

- The difference in the deviations between the five repeated measurement values was decreased by reducing the radius of the stylus tip.

- Minimum deviation belongs to stylus tip with the radius of $2.5 \mathrm{~mm}$ at speeds of 10 and $15 \mathrm{~mm} / \mathrm{s}$, as well as to the tip radius of $1.5 \mathrm{~mm}$ at speeds of 5 and $10 \mathrm{~mm} / \mathrm{s}$ only.

It is quite clear that the repeatability error is smaller when applying limited stylus tip through the CMM. This suggests that machine errors are affecting the results of the probing system itself, due to the fact that CMM probe system is being sensitive to the stylus tip, it may be due to the hysteresis effects mainly caused by friction at the seating contact which are magnified by elastic deflection of the stylus. There are obviously some dissimilarities which could be attributed strongly to the probe tip bending effects, length and the mass of the stylus beside its tip radius geometry.

\section{ANALYSIS OF THE OBTAINED UNCERTAINTY}

Estimation of the uncertainty of waviness measurements for the circler feature is a very complex undertaking, where the uncertainty should be based on many different factors and procedural steps of experimental and statistical techniques. There are different recognized techniques for determining the uncertainty of coordinate measurements made with CMMs [27].

Regression and variance for experimental data analysis are applied. Table 3 shows the statistical determination of the values of $M_{A V}, S_{D}$ and $U_{A} . M_{A V}$ is the mean average value of five repeated test measurements, $S_{D}$ is the standard deviation, and $U_{A}$ represents the uncertainty (type A) due to measurement repeatability. The number $n$ is 5 repeating test measurements, and $x_{i}$ is the measured value in roundness waviness, $\mu \mathrm{m}$. Fig. 8 shows the uncertainty of repeatability for the given tip sizes and scanning speeds. It ensures that the largest probe stylus tip has the highest level of uncertainty (type A) within the low measurement speed range from 5 to $15 \mathrm{~mm} / \mathrm{s}$, while it generates a relatively low uncertainty level at $25 \mathrm{~mm} / \mathrm{s}$. The maximum level of uncertainty (type A) belongs to the smallest stylus tip, especially at speed of $20 \mathrm{~mm} / \mathrm{s}$. The uncertainty has moderate values with the tip radius of $2.5 \mathrm{~mm}$ compared to the others at most speeds.

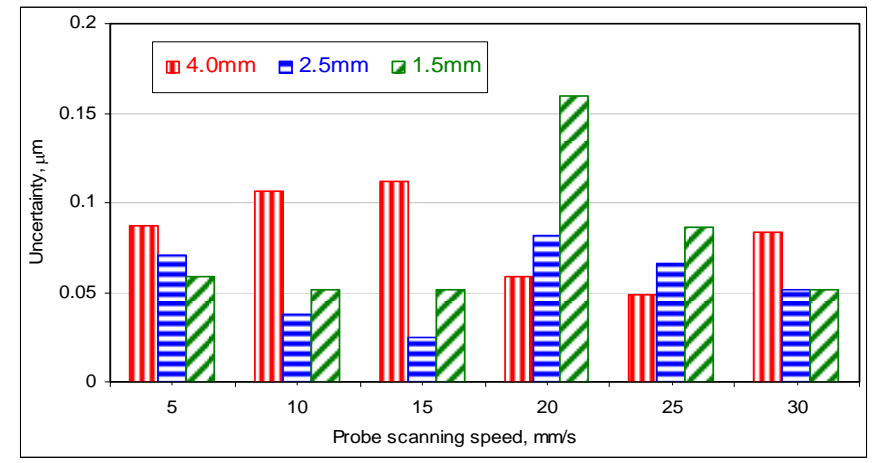

Fig.8. Uncertainty (Type A) of roundness form error for probe radii at different scanning speeds.

Table 3 Uncertainty (type A) of three probe stylus tip sizes at different scanning speeds.

\begin{tabular}{|c|c|c|c|c|c|c|c|c|c|}
\hline \multirow{3}{*}{$\begin{array}{c}\text { Stylus tip } \\
\text { scanning } \\
\text { speed, mm/s }\end{array}$} & \multicolumn{9}{|c|}{ Stylus tip radius, $\mathrm{mm}$} \\
\hline & \multicolumn{3}{|c|}{4.0} & \multicolumn{3}{|c|}{2.5} & \multicolumn{3}{|c|}{1.5} \\
\hline & $\mathrm{M}_{\mathrm{AV}}$ & $\mathrm{S}_{\mathrm{D}}$ & $\mathrm{U}_{\text {Type }}$ & $\mathrm{M}_{\mathrm{AV}}$ & $\mathrm{S}_{\mathrm{D}}$ & $\mathrm{U}_{\text {TypeA }}$ & $\mathrm{M}_{\mathrm{AV}}$ & $\mathrm{S}_{\mathrm{D}}$ & $\mathrm{U}_{\text {TypeA }}$ \\
\hline 5 & 2.46 & 0.19 & $\begin{array}{c}\mathrm{A} \\
0.09\end{array}$ & 2.60 & 0.16 & 0.07 & 2.92 & 0.13 & 0.06 \\
\hline 10 & 2.62 & 0.24 & 0.11 & 2.68 & 0.08 & 0.04 & 2.84 & 0.11 & 0.05 \\
\hline 15 & 2.74 & 0.25 & 0.11 & 2.76 & 0.05 & 0.02 & 2.96 & 0.11 & 0.05 \\
\hline 20 & 2.92 & 0.13 & 0.06 & 3.04 & 0.18 & 0.08 & 3.12 & 0.36 & 0.16 \\
\hline 25 & 2.92 & 0.11 & 0.05 & 3.08 & 0.15 & 0.07 & 3.52 & 0.19 & 0.09 \\
\hline 30 & 3.00 & 0.19 & 0.08 & 3.04 & 0.11 & 0.05 & 3.26 & 0.11 & 0.05 \\
\hline
\end{tabular}




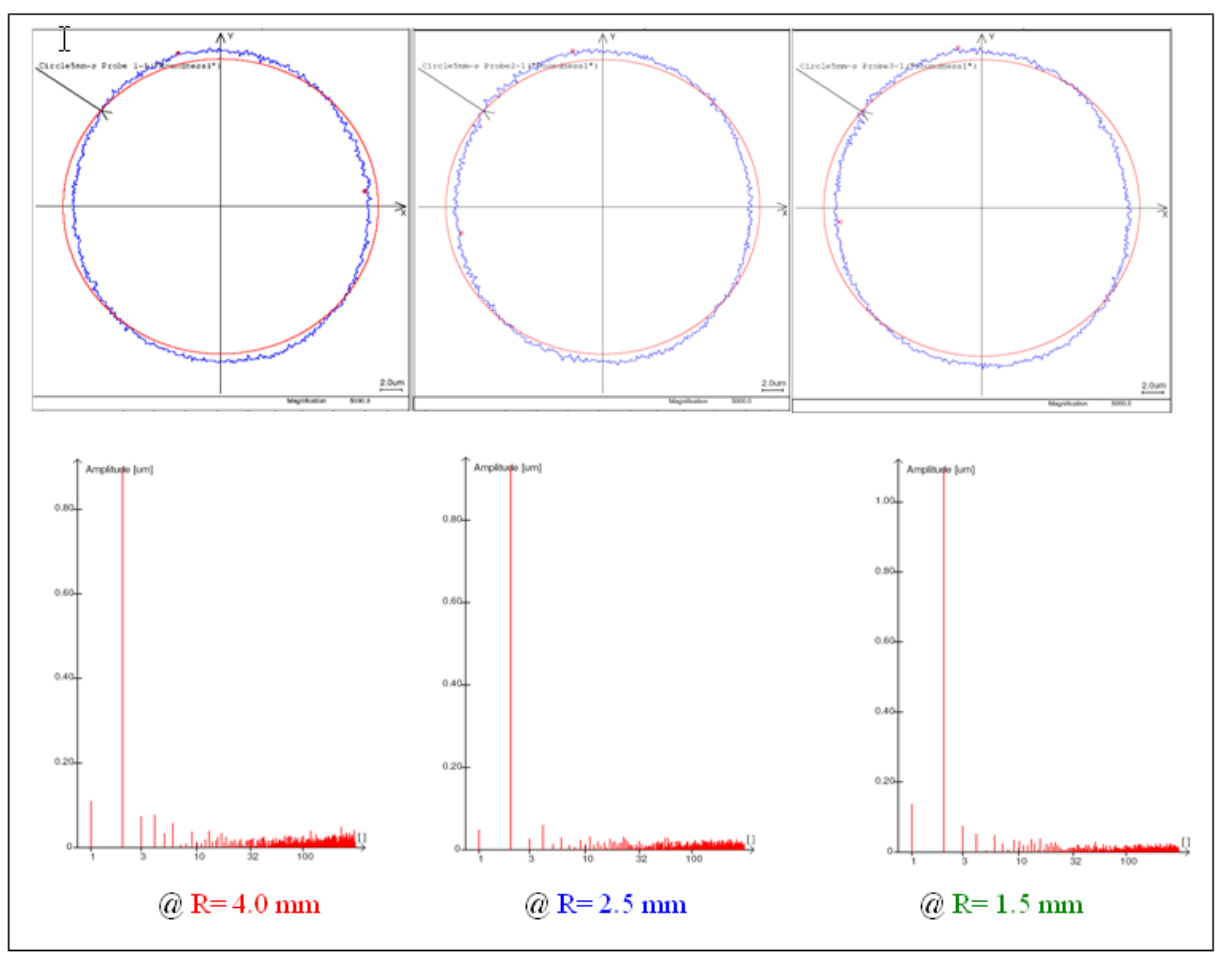

Fig.9. Profile waviness accuracy at different probe tip sizes in the time and frequency domains.

\section{EXPERIMENTAL RESULTS AND DISCUSSION}

Each experimental measurement provided two compatible sets of collected data. The first set is the selection of probe ball tips including their specifications of radii, stylus mass, stylus length and the probing scanning speeds related to cylindrical standard artifact. While the second set contains the corrected data set, it uses the CMM built-in algorithm to signify the dynamic errors due to both probe stylus tip sizes and probe scanning speeds in the measurements. Figs.9 and 12 show the experimental results of some typical selective samples from CMM machine.

\subsection{Effect of Probe Stylus Tip Size}

Fig.9 shows the accuracy of waviness profile of response carried out signals of CMM machine in the NIS laboratory for three different probe tip radii at $5 \mathrm{~mm} / \mathrm{s}$ probe scanning speed in both time domain and in frequency domain using built-in fast Fourier transformer.

Signal analyses illustrate that carried out surface waviness has a significant response at $2 \mathrm{~Hz}$ effective frequency for all measured samples. The highest value $1.0952 \mu \mathrm{m}$ of surface frequency response belongs to the tip radius $1.5 \mathrm{~mm}$, while stylus ball tip radius $2.5 \mathrm{~mm}$ recorded the minimum resonance response of $0.9303 \mu \mathrm{m}$ with a decrement of about $17 \%$ of the averaged response for the same natural frequency. These detections are carried out on the same path at the same probing speed on the used artifact. Consequently, the frequency response at $2 \mathrm{~Hz}$ for the samples clarifies that this value is a typical natural frequency of the surface detection speed. Therefore, the $17 \%$ frequency response variation can be considered as a detection error of stylus characteristics, where the stylus tip radius $2.5 \mathrm{~mm}$ is the suitable one for these performed conditions at low signal noise within the measured frequency band.

The results presented in Fig.9 give a full consensus of the frequency response at the surface wave natural frequency of $2 \mathrm{~Hz}$ with the averaged error value for selected trigger probes at the same probing speed of $5 \mathrm{~mm} / \mathrm{s}$. It clarifies that affecting the stability of the sine wave that appeared at each of the used probes may be of significance due to the path stability of the measured surface of the used artifact, as an input source. The difference between frequency response peaks for different probes may represent the level of the impacts due to probe dynamic movements during the surface detection.

Measurement averaged errors decrease according to any increase in stylus tip radius or probe detection speed, as shown in Fig.10. The error values lie within the range from about $3.5 \mu \mathrm{m}$ to about $2.5 \mu \mathrm{m}$, the greater errors belong to stylus tip radius $1.5 \mathrm{~mm}$. It can be notice that the averaged value of the waviness form deviation slope decreased significantly for stylus tip of $1.5 \mathrm{~mm}$ measurements at detection speed of $25 \mathrm{~mm} / \mathrm{s}$, it may be due to the influence of this speed on the resonance of the CMM machine elements. The averaged errors have significant decreased levels with the tip radius $4.0 \mathrm{~mm}$; it may be due to low dynamic response far away from CMM machine elements critical speeds, especially at $5 \mathrm{~mm} / \mathrm{s}$ speed.

In other words, Figs.10-11 can help to conclude that the small probe tip of $1.5 \mathrm{~mm}$ is better for diagnosing the true state of the surface form of the specimens than a bigger tip radius of 2.5 and $4.0 \mathrm{~mm}$, respectively. This is because the probe tips of the large radii possess a large contact area with the inner surface of the used standard artifact, and vice 
versa. In this case, the distortion of the measurement result using $1.5 \mathrm{~mm}$ probe tip becomes more visible and gives better estimate of the measured feature profile compared to the results of $4.0 \mathrm{~mm}$ probe tip.

Fig.12 shows measuring of 6 samples on the CMM machine at different probing scanning speeds in the laboratory. It presents the accuracy of waviness profile of typical measured results at different probe tip scanning speeds of the largest probe tip of $4.0 \mathrm{~mm}$ at magnification ratio of $5000: 1$. The effects of probing speed on the roundness error averaged values for different probe tip radii are detailed in Fig.13.

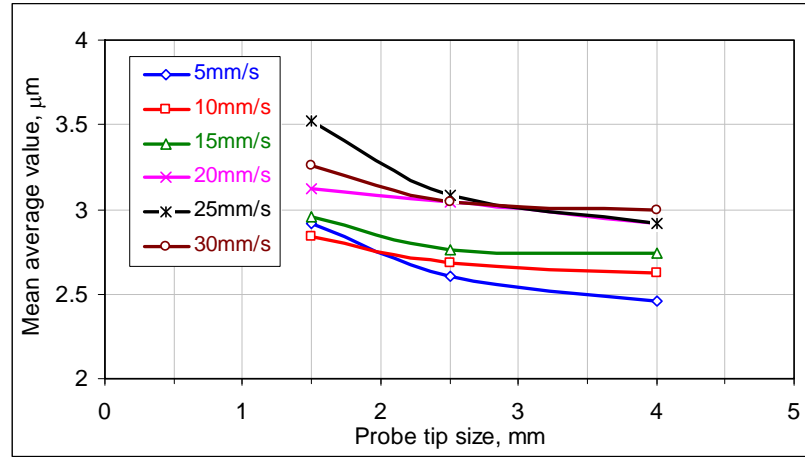

Fig.10. Effects of stylus tip radius size on the form error mean values at different probing speeds.

\subsection{Effect of Probing speed}

Variations of the probing scanning speed range from 5 to $30 \mathrm{~mm} / \mathrm{s}$ have a growing rate of averaged errors of sample measurements for all probe tip radii, it may be due to impact rate of surface asperities with the tip at high scanning speeds, Fig.13. The smallest tip radius generates high rates of averaged error, more than the other tips due to frequent impacts of relatively small asperities at various contact points of the standard artifact surface topography during the measuring process, which confirmed its high accuracy of surface roughness diagnosis in accordance with the real tip contact area.

The averaged error value of the probe tip $2.5 \mathrm{~mm}$ lies between 2.6 to $3.1 \mu \mathrm{m}$ with a magnitude of about $0.5 \mu \mathrm{m}$, while small probe tip with radius $1.5 \mathrm{~mm}$ lies within the range 2.4 to $3.0 \mu \mathrm{m}$ with $0.6 \mu \mathrm{m}$ variation error value. The large tip radius $4.0 \mathrm{~mm}$ has an error range between 2.8 to 3.5 $\mu \mathrm{m}$ with varying disbursement of $0.7 \mu \mathrm{m}$. The maximum deviations of the relative measured errors for both 1.5 and 4 $\mathrm{mm}$ tip radii grow from $10 \%$ for scan speed $15 \mathrm{~mm} / \mathrm{s}$ to reach about $17 \%$ corresponding to scan speeds of 5 , and 25 $\mathrm{mm} / \mathrm{s}$.

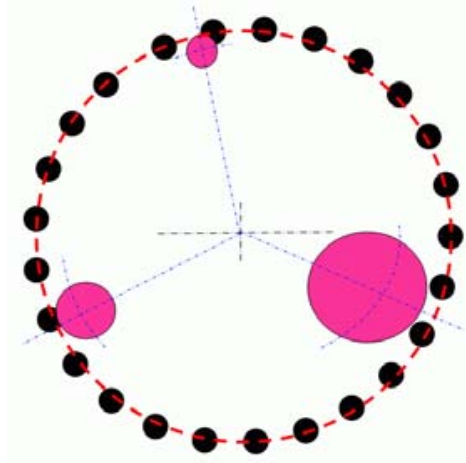

Fig.11. Scheme of the probe tips scanning path during measurement.

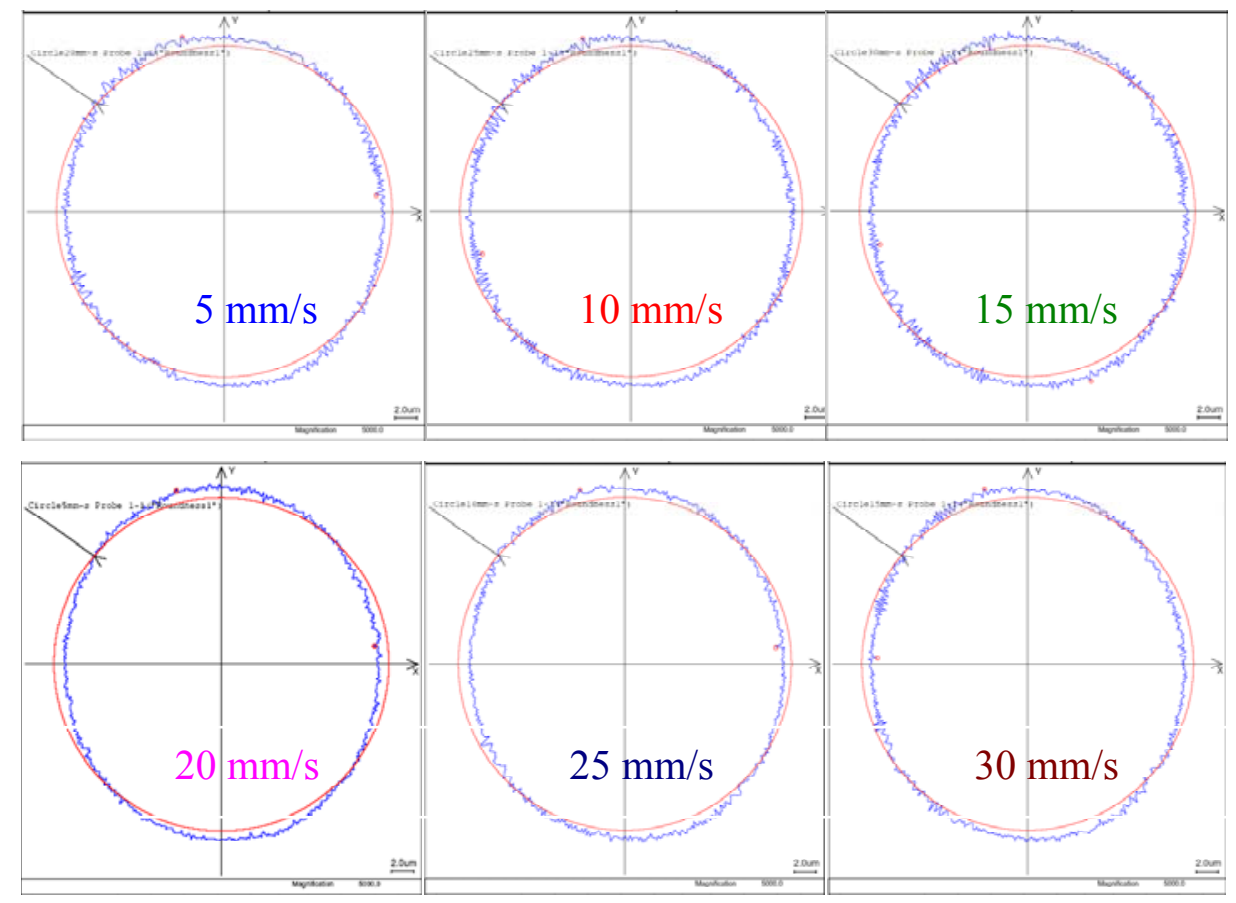

Fig.12. Sample response of $4.0 \mathrm{~mm}$ probe tip at different scanning speeds. 


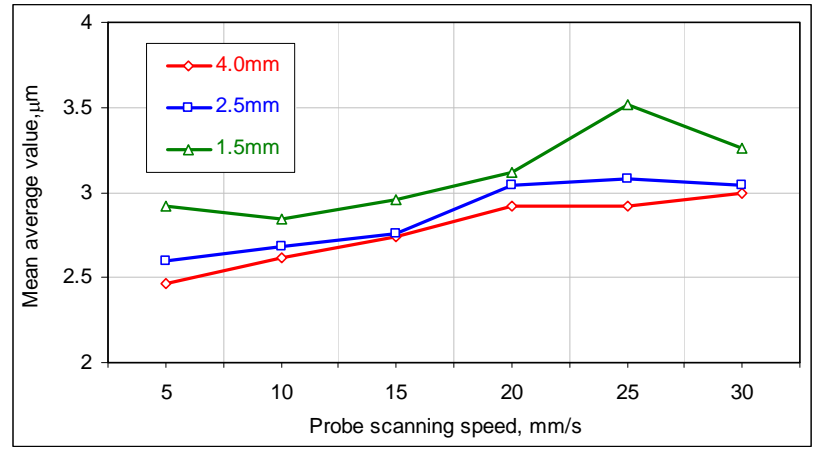

Fig.13. Effects of probe scanning speed on the roundness averaged error for different tips.

\section{CONCLUSIONS}

Findings of this study can be divided into two categories.

1-From the analytical result analysis, it can be concluded that:

- The proposed mathematical 2DM proved to be capable of clearly viewing two different systematic errors with the movement of the probe during scanning. The first error always occurs due to swerving of the ball tip as it rotates during the scanning process on the measured surface. This error is presented in the Y-direction, with zero value where the tip begins to start rotation and reach its maximum value of $2 \mathrm{R} \%$ at the point of orthogonal axis at $180^{\circ}$, then returns again to zero error at $360^{\circ}$. The second systematic error results from increasing the radius of the probe styli ruby ball tips.

2-From carried out results analysis, it can be concluded that:

- Increasing the probe tip radius decreases the averaged measured error signals of surface waviness. This may be attributed to the resulted reduced number of contact points as compared with those of smaller tip on the same artifact surface along the scanning trajectory.

- The amplitude of average error in the waviness profile increased with the probing scanning speed within the range of application. This may be attributed to the vibration excitation of the probe head as it scans the surface. It has become evident that the probe stylus tip and probe scanning speed have a significant influence on the accuracy of CMM measurements using the strategy of touch trigger probe independently.

- Based on the results, easy calibration and correction techniques can be established for probe performance accuracy of CMMs measurements. These techniques can be formulated with relevance to geometrical surface form and probe stylus dynamic characteristics.

\section{REFERENCES}

[1] Weckenmann, A., Estler, T., Peggs, G., McMurtry, D. (2004). Probing systems in dimensional metrology. CIRP Annals-Manufacturing Technology, 53 (2), 657-684.
[2] Woźniak, A., Dobosz, M. (2005). Influence of measured objects parameters on CMM touch trigger probe accuracy of probing. Precision Engineering, 29 (3), 290-297.

[3] Woźniak, A., Mayer, J.R.R., Bałaziński, M. (2009). Stylus tip envelop method: corrected measured point determination in high definition coordinate metrolog. Int. Journal of Advanced Manufacturing Technology, 42, 505-514.

[4] Wu, Y., Liu, S., Zhang, G. (2004). Improvement of coordinate measuring machine probing accessibility. Precision Engineering, 28 (1), 89-94.

[5] Park, J.-J., Kwon, K., Cho, N. (2006). Development of a coordinate measuring machine (CMM) touch probe using a multi-axis force sensor. Measurement Science and Technology, 17, 2380-2386.

[6] Hermann, G. (2007). Geometric error correction in coordinate measurement. Acta Polytechnica Hungarica, 4 (1), 47-62.

[7] Schwenke, H., Knapp, W., Haitjema, H., Weckenmann, A., Schmitt, R., Delbressine, F. (2008). Geometric error measurement and compensation of machines-an update. CIRP Annals-Manufacturing Technology, 57, 660-675.

[8] Genest, D.H. (1997). The right probe system adds versatility to CMMs. Quality Digest, http://www.qualitydigest.com/jan97/probes.html

[9] Dobosz, M., Woźniak, A. (2005). CMM touch trigger probes testing using a reference axis. Precision Engineering, 29 (3), 281-289.

[10] Zeiss Calypso Navigator (2004). CMM Operation Instructions and Training Manual. Revision 4.0. Oberkochen, Germany: Carl Zeiss Co.

[11] Ali, S.H.R. (2008) The Influence of fitting algorithm and scanning speed on roundness error for $50 \mathrm{~mm}$ standard ring measurement using CMM. Journal of Metrology \& Measurement Systems, 15 (1), 31-53.

[12] Kasparaitis, A., Šukys, A. (2006). Dynamic errors of CMM probes. Solid State Phenomena: Mechatronic Systems and Materials, 113, 477-482.

[13] Ali, S.H.R, Mohamed, H.H., Bedewy, M.K. (2009). Identifying cylinder liner wear using precise coordinate measurements. Int. Journal of Precision Engineering and Manufacturing, 10 (5), 19-25.

[14] Lin, Y.C., Sun, W.I. (2003). Probe radius compensated by the multi-cross product method in free form surface measurement with touch trigger probe CMM. Int. Journal of Advanced Manufacturing Technology, 21, 902-909.

[15] Li, L., Jung, J.-Y., Lee, CH.-M., Chung, W.-J. (2003). Compensation of probe radius in measuring freeformed curves and surface. Int. Journal of the Korean Society of Precision Engineering, 4 (3).

[16] Xiong, Z., Li, Z. (2003). Probe radius compensation of workpiece localization. Transactions of the ASME, 125, 100-104.

[17] Ali, S.H.R. (2010). Two dimensional model of CMM probing system. Journal of Automation, Mobile Robotics \& Intelligent Systems, 4 (2), 3-7. 
[18] Sokovic, M., Cedilnik, M., Kopac, J. (2007). Identification of scanning errors using touch trigger probe head. Journal of Achievements in Materials and Manufacturing Engineering, 20 (1-2), 383-386.

[19] Estler, W.T., Phillips, S.D., Borchardt, B., Hopp, T., Witzgall, C., Levenson, M., Eberhardt, K., McClain, M., Shen, Y., Zhang, X. (1996). Error compensation for CMM touch trigger probes. Precision Engineering, 19 (2), 85-97.

[20] Pereira, P.H., Hocken, R.J. (2007). Characterization and compensation of dynamic errors of a scanning coordinate measuring machine. Precision Engineering, 31(1), 22-32.

[21] Hessling, J.P. (2008). Dynamic metrology - an approach to dynamic evaluation of linear timeinvariant measurement systems. Measurement Science and Technology, 19 (8).

[22] Farooqui, A., Morse, P. (2007). Methods and artifacts for comparison of scanning CMM performance. Transactions of the ASME, 7, 72-80.
[23] Kosarevsky, S., Latypov, V. (2010). Inertia compensation while scanning screw threads on coordinate measurement machines. Measurement Science Review, 10 (2), 68-71.

[24] Yagüe, J.-A., Albajez, J.-A., Velázquez, J., Aguilar, J.J. (2009). A new out-of-machine calibration technique for passive contact analog probes. Measurement, 42, 346-357.

[25] Zhao, J., Fei, Y.T., Chen, X.H., Wang, H.T. (2005). Research on high-speed measurement accuracy of coordinate measuring machines. Journal of Physics: Conference Series, 13, 167-170.

[26] Geometrical product specifications (GPS) Acceptance and reverification tests for coordinate measuring machines (CMM) - Part 2: CMMs used for measuring linear dimensions. (2009). Standard ISO 10360-2.

[27] Beaman, J., Morse, E. (2010). Experimental evaluation of software estimates of task specific measurement uncertainty for CMMs. Precision Engineering, 34, 28-33. 